\title{
Globalización y
}

\section{competencia tributaria: implicaciones para los países en desarrollo}

\section{Reuven S. Avi-Yonah}

Facultad de Derecho de la Universidad de Michigan
Este artículo analiza el efecto de la competencia tributaria sobre los países en desarrollo. Como resultado de la globalización y de la mayor movilidad del capital, muchos países en desarrollo han adoptado a partir del decenio de 1980 la política de competir unos con otros por atraer inversión de capital. Una de las formas principales que toma esta competencia es la de otorgar franquicias tributarias y otras reducciones de gravámenes a las empresas transnacionales inversionistas. En el presente trabajo se pasa revista a los argumentos normativos a favor y en contra de esta forma de competencia tributaria, desde una perspectiva global. En seguida se examinan a fondo esos argumentos desde el punto de vista de los países en desarrollo. En general, se concluye que, puesto que las empresas transnacionales invertirían en países en desarrollo sin recibir subsidios tributarios, pero que tienen la posibilidad de recibirlos a través de una suerte de licitación entre los países en desarrollo, a estos países les convendría más concordar en abstenerse de otorgar tales subsidios. Por último, se analizan algunas vías para lograr una cooperación de este tipo, ya sea regional o global (por ejemplo, a través de la Organización Mundial del Comercio). 


\section{Introducción}

La actual era de la globalización puede distinguirse de la anterior (la de 1870 a 1914), porque en la primera la movilidad del capital es mucho mayor que la del trabajo (en la era anterior, antes de las restricciones inmigratorias, la fuerza de trabajo era por lo menos tan móvil como el capital). Esta mayor movilidad del capital obedece a los cambios tecnológicos (la capacidad de traspasar fondos electrónicamente) y a la distensión de los controles cambiarios. Debido a la movilidad del capital, los países soberanos reducen los impuestos con que gravan la renta percibida por extranjeros dentro de sus fronteras, con el fin de atraer inversiones de cartera y directas. Es lo que se ha denominado competencia tributaria. A su vez, esta competencia tributaria amenaza con socavar los impuestos que gravan los ingresos de las personas físicas y las utilidades de las empresas y que, por el porcentaje del total de los ingresos fiscales que representan, constituyen importantes fuentes de recursos para todos los Estados modernos. La primera reacción de los países desarrollados y en desarrollo ante el fenómeno descrito ha sido trasladar la carga tributaria del capital (móvil) a la mano de obra (menos móvil) y, posteriormente, cuando política y económicamente se ha tornado difícil aumentar más la tributación sobre la mano de obra, recortar los servicios públicos. Así, pues, la globalización y la competencia tributaria llevan a una crisis fiscal a los países que desean seguir prestando esos servicios a sus ciudadanos, en tanto que los factores demográficos y el aumento de la desigualdad y volatilidad de los ingresos y de la inseguridad en el empleo que se derivan de la globalización hacen más necesarios dichos servicios. En el presente trabajo se sostiene que, para mantener los programas de servicios públicos frente a la globalización, es preciso cortar el vínculo intermedio, restringiendo la competencia tributaria. Sin embargo, por consideraciones prácticas y normativas, los límites establecidos a tal competencia deben ser congruentes con el mantenimiento de la capacidad de los Estados democráticos de decidir cuál ha de ser el tamaño de sus gobiernos.

\section{II}

\section{La competencia tributaria internacional y los impuestos sobre el capital}

Desde sus inicios a fines del siglo XIX, el Estado moderno se ha financiado principalmente con un gravamen progresivo sobre los ingresos. Este impuesto difiere de otras formas de tributación (como los impuestos sobre el consumo o de seguridad social) en que, teóricamente, en la base tributaria se incluye el ingreso del capital, aunque éste haya sido ahorrado y no consumido.

Como los ricos ahorran más que los pobres, un impuesto que incluye los ingresos de capital en su base es más progresivo (grava más a los ricos) que un im-

\footnotetext{
$\square$ Este trabajo fue encomendado al autor por la División Fiscal del Departamento de Integración y Programas Regionales del Banco Interamericano de Desarrollo (BID). Las opiniones que se expresan en él no reflejan necesariamente las de esa institución.
}

puesto que los excluye (por ejemplo, aquél sobre el consumo o sobre los salarios). No obstante, la capacidad de gravar los ingresos de capital ahorrados (por ejemplo, ingresos no sujetos a los impuestos sobre el consumo) se ve reducida si el capital puede trasladarse al extranjero, a jurisdicciones donde escapa a la tributación.

Recientemente se han producido dos hechos que han aumentado marcadamente la capacidad de individuos y empresas de no pagar impuestos por sus ingresos percibidos en el extranjero: el fin definitivo de los impuestos de retención sobre los pagos de intereses en los países desarrollados, y el aumento de los paraísos tributarios "para la producción" en los países en desarrollo (Avi-Yonah, 2000). Desde que los Estados Unidos abolió su práctica de aplicar impuestos de reten- 
ción sobre los pagos de intereses a extranjeros en 1984, ningún país que sea un importador de capital importante ha podido imponer dicho tributo por miedo a ahuyentar el capital móvil hacia otra parte, o elevar el costo del capital para los prestatarios nacionales, incluido el propio gobierno (Tanzi, 1995; Gardner, 1992). Por consiguiente, en general los individuos pueden percibir, libres de impuestos del país huésped, ingresos provenientes de inversiones en cualquiera de las principales economías del mundo (Avi-Yonah y Swartz, 1997; Cohen, 1998; May, 1996). Además, aun para los países desarrollados resulta extremadamente difícil recaudar con eficacia el impuesto sobre los ingresos en el extranjero de sus residentes si el país huésped no retiene impuestos, ya que las inversiones pueden hacerse a través de paraísos tributarios con leyes estrictas de protección del secreto bancario (Tanzi, 1995). A los países en desarrollo, con administraciones tributarias mucho más débiles, esta tarea les resulta casi imposible. De manera que, en gran medida, los ingresos por inversiones transfronterizas pueden obtenerse libres de impuestos tanto del país huésped como del país de origen (Kant, 1996; McLure, 1989).

Por ejemplo, tomemos el caso de un mexicano adinerado que desea ganar intereses libres de impuestos con sus inversiones en bonos de una empresa estadounidense. Todo lo que tiene que hacer es crear, por una suma nominal, una empresa en las Islas Caimán para que ella sea la titular de los bonos. Luego los pagos de intereses se hacen a esta empresa, sin que se retenga impuesto estadounidense alguno, en virtud de la denominada exención del pago de intereses procedentes de valores de inversión (Código de Impuestos Internos de los Estados Unidos, sección 871-h). La persona no informa del ingreso percibido a las autoridades mexicanas, y éstas no tienen manera de saber que la empresa de las Islas Caimán es en realidad una empresa ficticia del residente mexicano. Tampoco ayudan las disposiciones sobre intercambio de información del acuerdo tributario vigente entre los Estados Unidos y México, dado que el Servicio de Impuestos Internos de los Estados Unidos no tiene cómo saber que la empresa que percibe los intereses está bajo el control de un residente mexicano y, por lo tanto, no puede informar de ello a las autoridades mexicanas. En consecuencia, los ingresos quedan totalmente libres de impuestos (por cierto, las Islas Caimán no cobra impuestos sobre la renta propios).

Si dirigimos nuestra atención a la inversión productiva más que a la pasiva, veremos que surge allí una amenaza semejante a la capacidad impositiva de las jurisdicciones del país huésped y del país de origen. En la década de 1990, la competencia por atraer inversión externa ha llevado a un número cada vez mayor de países (103 en 1998) a ofrecer franquicias tributarias destinadas específicamente a empresas inversionistas extranjeras (Vernon, 1998; unCTAD, 1996). Dada la relativa facilidad con que una empresa transnacional integrada puede trasladar sus instalaciones productivas en respuesta a las tasas de impuestos, tales paraísos tributarios "para la producción" permiten que las transnacionales perciban la mayor parte de sus ingresos del extranjero libres de los impuestos del país huésped (Hines y Rice, 1994; Altshuler y Newlon, 1993). Además, en su mayoría, los países desarrollados (incluidos los Estados Unidos) no se atreven a cobrar los impuestos vigentes (y a veces ningún impuesto) sobre los ingresos comerciales en el extranjero de sus empresas transnacionales residentes, por temor a reducir la competitividad de estas empresas frente a las de otros países (Peroni, 1997). Si lo hicieran, se podrían crear nuevas transnacionales como residentes de jurisdicciones que no gravan tales ingresos (Hines, 1991). Así, los ingresos de las empresas también pueden percibirse en el extranjero libres de impuestos tanto del país huésped como del país de origen.

Por ejemplo, la Intel Corporation, empresa transnacional que figura entre las primeras 10 del mundo, opera en más de 30 países. Esta empresa dice que un chip que se diseña en Oregón puede fabricarse en una planta en Irlanda, embalarse y probarse en Malasia y luego venderse a un cliente en Australia. Otra puede diseñarse en Japón, fabricarse en Israel, embalarse y probarse en Arizona y venderse en China (Intel Corporation, 1998). Concretamente, fuera de los Estados Unidos la Intel tiene grandes fábricas en Puerto Rico, China, Malasia, Filipinas, Irlanda e Israel (Intel Corporation, 1999). Por lo tanto, salvo las ubicadas en los Estados Unidos, todas se hallan en países que otorgan franquicias tributarias. Intel tampoco paga los impuestos vigentes en los Estados Unidos sobre sus ingresos procedentes de tales operaciones en el extranjero, dado que, según las leyes estadounidenses, el ingreso activo percibido por filiales extranjeras de transnacionales estadounidenses sólo está sujeto a impuesto cuando vuelve al país en forma de dividendos, lo que Intel puede postergar por muchos años (Avi-Yonah, 1997). De manera que la verdadera tasa impositiva sobre los ingresos de Intel procedentes del extranjero está muy por debajo de la tasa nominal de 35\% vigente en los Estados Unidos. 
Si los ingresos del capital pueden evadir el impuesto sobre los ingresos, éste pasa a ser de hecho un impuesto sobre el trabajo. En efecto, varios estudios empíricos han insinuado que en algunas jurisdicciones de países desarrollados la tasa impositiva efectiva sobre el ingreso del capital se acerca a cero, y los impuestos sobre el capital han tendido a reducirse marcadamente desde principios del decenio de 1980, cuando se relajaron los controles cambiarios (Owens y Sasseville, 1997; Rodrik, 1997). En consecuencia, los países que solían recurrir a las recaudaciones procedentes del impuesto sobre los ingresos han tenido que elevar impuestos que son relativamente regresivos. Los dos impuestos que han aumentado con más rapidez en los países miembros de la OCDE en los últimos años han sido aquéllos sobre el consumo (del 12\% al 18\% de la recaudación total entre 1965 y 1995) y sobre la nómina (del 19\% al 27\%); ambos son más regresivos que el impuesto sobre los ingresos (Owens y Sasseville, 1997). En el mismo período, los tributos sobre el ingreso de las personas y sobre las utilidades de las empresas no se incrementaron como porcentaje de la recaudación: el impuesto sobre el ingreso personal alcanzó al 26\% de ella en 1965 y al $27 \%$ en 1995, en tanto que aquél sobre las utilidades de las empresas llegó al 9\% y al 8\%, respectivamente (Owens y Sasseville, 1997). El total de ingresos tributarios como porcenta- je del PIB en los países desarrollados se disparó en el mismo período (de un promedio de $28 \%$ en 1965 a casi $40 \%$ en 1994); este incremento obedeció, en gran medida, al alza de los impuestos sobre el consumo y sobre los salarios (Banco Mundial, 1994). Además, hay indicios en los países miembros de la OCDE de que, a medida que aumenta la apertura de una economía, los impuestos sobre el capital tienden a bajar y aquéllos sobre el trabajo a subir; como el impuesto sobre los ingresos grava tanto el capital como la mano de obra, su estabilidad bien podría encubrir esta tendencia (Mendoza, Razin y Tesar, 1994; Mendoza, MilesiFerretti y Asea, 1996).

Las mismas tendencias pueden observarse en los países en desarrollo. En países que no son miembros de la OCDE, excluidos los del Oriente medio, el total de la recaudación pública como porcentaje del PIB subió de un promedio de $18.8 \%$ en $1975-1980$ a uno de $20.1 \%$ en 1986-1992 (Banco Mundial, 1994). Este aumento se financió, sobre todo, con el crecimiento de la recaudación procedente del impuesto al valor agregado en el mismo período (de $25.5 \%$ del total al $31.8 \%$ ). Al mismo tiempo, los ingresos provenientes del impuesto sobre el ingreso de las personas y sobre las utilidades de las empresas permanecieron estables o bajaron (Banco Mundial, 1994).

\section{III}

\section{La competencia tributaria y los países en desarrollo}

Las desventajas de la competencia tributaria para los países desarrollados son relativamente claras, dado que ella amenaza el alto nivel de gasto público que su compleja red de seguridad social exige (Leibfritz y otros, 1995). Pero veamos en qué forma dicha competencia afecta a los países en desarrollo.

En primer lugar, cabe señalar que los países en desarrollo necesitan ingresos por lo menos en la misma medida que los países desarrollados, si no más. Suele pensarse erróneamente que sólo los países de la OCDE sufren una crisis fiscal por el aumento de las personas de edad en su población. De hecho, se prevé que la relación de dependencia (entre las personas de edad y la población que trabaja) también ha de aumen- tar en otras regiones geográficas, a medida que se reduzcan las tasas de fecundidad y mejore la atención de la salud (Banco Mundial, 1994). Fuera de las economías en transición y de la OCDE, la relación de dependencia empezó exhibiendo cifras de un dígito en la década de 1990, pero aumentaría a algo menos de 30\% hacia el 2100 (McLure, 1996). Además, si bien fuera de las economías en transición y de la OCDE el gasto directo en seguro social es muy inferior, hay otras formas de gasto público (por ejemplo, el empleo público) que en efecto cumplen una función de seguro social. En América Latina, por ejemplo, el gasto público directo en seguro social es muy inferior al gasto indirecto a través del empleo público y los pro- 
gramas de adquisiciones del gobierno (Subbarao y otros, 1997).

Por lo demás, parece inadecuado sostener que los países en desarrollo necesitan de los ingresos tributarios en menor medida que los países desarrollados, porque tienen programas menos avanzados de seguro social. A juzgar por el caso del seguro social, esa necesidad es aun mayor en los países en desarrollo debido a la pobreza generalizada, la que hace que la pérdida de un empleo pueda tener consecuencias mucho más nefastas (PNUD, 1997). Sin embargo, la necesidad de recaudaciones tributarias en los países en desarrollo va más allá del tema del seguro social. En algunos de ellos tales recaudaciones son necesarias para garantizar la existencia misma del gobierno organizado, como lo demuestra lo sucedido en Rusia (The Economist, 1998). En otros países más estables se precisan, principalmente, para proporcionar educación adecuada (inversión en capital humano), lo que muchos consideran clave para promover el desarrollo (Sen, 1997). Por ejemplo, las Naciones Unidas ha estimado que con sólo 30 a 40 mil millones de dólares la población de todo el mundo podría obtener servicios sociales básicos, como la enseñanza primaria (PNUD, 1997). Dadas las actuales tendencias en materia de ayuda externa, la mayoría de estos fondos tendría que provenir de gobiernos de países en desarrollo (Naciones Unidas, 2001).

En segundo lugar, los economistas habitualmente recomiendan a las pequeñas economías abiertas que no cobren impuestos a los inversionistas extranjeros, dado que no se les podría obligar a soportar la carga de un gravamen establecido por el país importador de capital (Razin y Sadka, 1991). El impuesto, por lo tanto, tendrá que aplicarse a factores menos móviles del país huésped, como la mano de obra y/o la tierra, y es más eficiente gravar a dichos factores directamente. No obstante, si bien este argumento parece válido para las inversiones de cartera, no tiene la misma validez ante la inversión extranjera directa (IED) por dos razones. La primera es que la recomendación no se aplica si el país de origen del inversionista efectúa deducciones tributarias por pago de impuestos en el extranjero, lo que sucede con frecuencia en el caso de la IED (Viherkentta, 1991). La segunda es que tal recomendación presupone que el país huésped es pequeño; sin embargo, la extensa literatura sobre empresas transnacionales sugiere que, en general, ellas existen para obtener rentas económicas (Hennart, 1991), en cuyo caso el país huésped deja de ser "pequeño" en el sentido económico. Es decir, hay razones para que el inversionista esté allí y no en otro lado. Por consiguien- te, el impuesto sobre esas rentas (siempre que sea inferior al 100\%) no ahuyentará necesariamente al inversionista, aunque éste no pueda traspasar la carga tributaria a la mano de obra o a los terratenientes.

Claramente, este argumento es válido cuando se trata de ingresos vinculados a una ubicación específica, como en el caso de los recursos naturales o un mercado de gran envergadura. Pero, ¿qué ocurre si el ingreso se puede obtener en un gran número de ubicaciones posibles (Dunning, 1988)? En este caso, el país huésped no podrá gravarlo si la empresa transnacional amenaza seriamente con irse a otra parte, aunque una vez que la inversión se ha hecho, la renta es imponible. Ante esta situación, que es probablemente la más común (Hennart, 1991), haría falta tomar medidas coordinadas para permitir que todos los países huéspedes gravasen la renta obtenida dentro de sus fronteras.

Y aquí llegamos al argumento final: que los países huéspedes deben ofrecer incentivos tributarios para ser competitivos. En gran cantidad de publicaciones se ha demostrado que de hecho los impuestos tienen una incidencia crucial al decidir la localización de las inversiones (Bond, 1981; Boskin y Gale, 1987; Hines, 1999). Sin embargo, en todos esos estudios se recalca que los incentivos tributarios son cruciales si se dispone de tales incentivos en otros lugares (Guisinger y otros, 1985). Así, se podría decir que, dada la necesidad de recaudar impuestos, en general los países en desarrollo preferirían no otorgar incentivos tributarios, si sólo se les pudiera garantizar que los demás países en desarrollo tampoco los otorgarían (Avi-Yonah, 2000).

En consecuencia, restringir la capacidad de los países en desarrollo de competir en el otorgamiento de incentivos tributarios no restringe realmente su autonomía ni va en contra de sus intereses. Es lo que sí ocurre cuando esos países otorgan el incentivo sólo por temor a la competencia de otros países en desarrollo. Cuando es la competencia de otros países lo que impulsa a establecer el incentivo tributario, eliminar dicha competencia no daña al país en desarrollo, y bien puede contribuir a sus esfuerzos por recaudar ingresos fiscales (suponiendo que pueda atraer inversiones por otros motivos, lo que generalmente ocurre). Además, según las propuestas que se describen a continuación, los países en desarrollo quedan libres de reducir sus impuestos en general (en lugar de otorgar un beneficio tributario específico a los inversionistas extranjeros).

Cabe hacer otras dos observaciones desde la perspectiva de los países en desarrollo. La primera tiene 
que ver con la incidencia de los impuestos. Como la competencia entre incentivos tributarios que tiene mayor importancia para los países en desarrollo se refiere al impuesto sobre las utilidades de las empresas, conviene tratar de determinar la incidencia de este impuesto al evaluar los efectos de su recaudación sobre el bienestar del país. Por desgracia, tras décadas de análisis, no existe consenso al respecto. Mientras que los estudios más antiguos han tendido a concluir que dicho impuesto recae sobre los accionistas o sobre todos los que aportan capital, los más recientes sugieren que es sufragado en grado considerable por los consumidores o por la fuerza de trabajo (Pechman, 1987; Estados Unidos, Departamento del Tesoro, 1992). También es posible que el impuesto sobre las empresas establecidas haya recaído en los que eran accionistas cuando el gravamen fue creado o incrementado, porque posteriormente fue capitalizado en el precio de las acciones (Pechman, 1987). Probablemente este debate no se resuelva en el futuro cercano (de hecho, es posible que la incidencia del impuesto esté cambiado con el tiempo, especialmente porque la globalización tal vez permita que las empresas traspasen una fracción mayor de la carga tributaria a la mano de obra). Sin embargo, desde la perspectiva de un país en desarrollo que debe decidir si cobrarle o no impuestos a una empresa transnacional, cabe considerar que tres de los cuatro grupos en que podría incidir el impuesto (los actuales accionistas o aportantes de capital, los antiguos accionistas y los consumidores) son en gran medida residentes de otras jurisdicciones y por consiguiente, desde una perspectiva de bienestar nacional, el país en desarrollo sale ganando si cobra el gravamen. Y aun en el caso de que cierta porción del impuesto se traspase a la fuerza laboral del país en desarrollo, se puede sostener que, en términos de administración tributaria, es más eficiente (y más aceptable políticamente) cobrarle el impuesto a una empresa transnacional que intentar cobrárselo a los trabajadores.

Por último, hay que señalar que el país en desarrollo puede querer cobrar impuestos a las empresas transnacionales, aunque crea que en general el sector privado utiliza los recursos con más eficiencia que el sector público. Esto porque, en el caso de una empresa transnacional de origen extranjero, los impuestos que el país en desarrollo no logra recaudar y que de hecho pueden ser utilizados por el sector privado, serán usados en otra jurisdicción y, por lo tanto, no beneficiarán al país. Una solución posible, que de hecho se aplica en los países en desarrollo, es no gravar a las transnacionales en tanto reinviertan en el ámbito nacional, y sí cobrarles impuestos cuando remitan sus utilidades al exterior. No obstante, esta tributación sobre dividendos y demás formas de remesas está sujeta al mismo problema de competencia tributaria que se trató anteriormente. Así, parecería que en la mayoría de los casos superar este problema es de interés para los países en desarrollo, y que lo que hay que decidir es cómo hacerlo frente a la necesidad de acción colectiva que acabamos de explicar.

\section{IV}

\section{¿Qué hacer respecto a la competencia tributaria?}

El problema de la competencia de esta índole es esencialmente uno de coordinación y confianza. Cada jurisdicción preferiría gravar a los inversionistas extranjeros con el fin de obtener ingresos, pero teme que al hacerlo los empujará a otras jurisdicciones donde no estén sujetos a impuestos. Si hubiera una manera de coordinar medidas entre jurisdicciones, todas podrían obtener ingresos adicionales sin correr el riesgo de perder inversión.

Esta dinámica queda bien ilustrada con la historia de la tributación alemana sobre los ingresos por concepto de intereses. En 1988, Alemania introdujo un impuesto de retención de $10 \%$ de los intereses devengados por los depósitos bancarios, pero tuvo que abolirlo después de pocos meses debido a que se produjo una enorme fuga de capitales a Luxemburgo. En 1991, el tribunal constitucional federal de Alemania dictaminó que la retención de impuestos sobre los salarios, pero no sobre los intereses, violaba el derecho constitucional a la igualdad, tras lo cual el gobierno reintrodujo la retención del impuesto sobre los intereses, pero la hizo inaplicable a los no residentes (Muten, 
1994). Sin embargo, los no residentes podían ser alemanes que invirtieran a través de cuentas bancarias en Luxemburgo. Para hacer frente a esta situación, los alemanes han encabezado una iniciativa de la Unión Europea tendiente a establecer un impuesto del 20\% sobre todos los pagos de intereses a sus residentes (Unión Europea, 1998). No obstante, hasta ahora Luxemburgo y el Reino Unido han bloqueado la aprobación de este plan, aduciendo que él provocaría una fuga de inversionistas a Suiza o los Estados Unidos (Annells, 1998).

De manera que la clave para encontrar una solución al problema de la competencia tributaria es atacarlo desde un frente multilateral amplio, a través de un ente como la Organización de Cooperación y Desarrollo Económicos (OCDE). En las actuales condiciones, la OCDE es la opción natural para encabezar las medidas coordinadas contra la competencia tributaria, por tres razones. Primero, porque para que los inversionistas obtengan un rendimiento razonable de su capital, sin incurrir en riesgos excesivos, deben invertir en un país miembro de la OCDE. Los paraísos tributarios no ofrecen suficientes oportunidades de inversión y, en general, los países en desarrollo se consideran muy riesgosos para la inversión de cartera (salvo los fondos mutuos, que no ofrecen oportunidades de elusión de impuestos). Así, si todos los países miembros de la OCDE gravaran la inversión de cartera, ésta podría estar sujeta a impuestos sin que fuese necesaria la colaboración de autoridades de los paraísos tributarios.

En segundo lugar, alrededor del $85 \%$ de las empresas transnacionales del mundo tienen su sede en países miembros de la OCDE, lo que seguramente continuará durante un tiempo, dado que dichos países ofrecen a los inversionistas la protección de leyes estables sobre empresas y valores que no están vigentes en otros países. Así, pues, si todos los países de la OCDE convinieran coordinadamente en cobrar impuestos a las empresas transnacionales sobre sus ingresos procedentes del extranjero, se podría resolver en gran parte el problema de competencia tributario en lo que toca a la inversión directa.

En tercer lugar, la OCDE cuenta con amplios conocimientos y experiencia en el tema (su acuerdo tributario modelo constituye el estándar mundial) y ya ha comenzado a limitar la competencia tributaria. En 1998 aprobó un informe titulado Harmful Tax Competition: An Emerging Global Issue (OCDE, 1998). Este informe es algo limitado, ya que sólo aborda la competencia tributaria relativa a las actividades y servicios financieros (y deja de lado, por ejemplo, las fábricas de
Intel); tampoco trata la tributación sobre los ingresos derivados de las inversiones. Pero representa un primer paso muy valioso y la prueba de que puede alcanzarse consenso en el tema de la competencia tributaria. (Suiza y Luxemburgo se abstuvieron en la aprobación de este informe, pero no se atrevieron a vetarlo en contra de los demás 27 miembros de la OCDE).

La OCDE hace una distinción útil entre la competencia tributaria que toma la forma de impuestos más bajos de aplicación general y los regímenes tributarios destinados a atraer inversionistas extranjeros. Esta distinción es sensata tanto desde el punto de vista normativo como pragmático. La restricción de la competencia tributaria no debe ni puede significar que los votantes de los países democráticos pierdan su derecho a determinar el tamaño del sector público a través de incrementos o reducciones generales de impuestos. Pero sí significa que los países no deben proporcionar ganancias inesperadas a los inversionistas extranjeros a costa de la capacidad de los demás países de prestar los servicios públicos que sus residentes desean. Tal restricción es particularmente apropiada, porque los propios inversionistas extranjeros suelen residir en países que prestan un alto nivel de servicios $\mathrm{y}$, sin embargo, se rehúsan a pagar el precio tributario que entraña prestarlos.

El hecho de recurrir a la OCDE para resolver el problema de la competencia tributaria tiene una gran desventaja: los países en desarrollo quedan fuera, y pueden estimar que las medidas adoptadas por la OCDE constituyen el accionar de un cartel de países ricos en contra de sus intereses. En efecto, como ya se indicó, no es probable que la competencia tributaria beneficie a los países en desarrollo, que también tienen necesidad de aquellos ingresos impositivos a los que renuncian para atraer inversionistas extranjeros. Si se pudiera impedir que todos los países en desarrollo compitieran de esta manera, todos ganarían. Pero a la larga, quizá sería mejor confiar la lucha contra la dañina competencia tributaria a la Organización Mundial del Comercio (OMC), donde los países en desarrollo están suficientemente representados. Así se resolvería también el problema de qué hacer con el 15\% de transnacionales cuya sede no está en los países de la OCDE (porcentaje que seguramente aumentará si en efecto esta organización toma medidas para restringir la competencia tributaria de sus transnacionales).

En suma, como resultado de la globalización y la competencia tributaria, la fijación de las reglas impositivas ya no puede estar a cargo de países que actúen unilateralmente ni mediante acuerdos tributarios 
bilaterales. En un mundo en que el capital puede moverse libremente a través de las fronteras nacionales y en que las transnacionales tienen libertad para elegir entre muchos sitios para localizar sus inversiones, es muy limitada la capacidad de un país (o de dos países en cooperación) de gravar dicho capital (o regularlo de alguna otra manera). Cualquier intento unilateral de ese tipo será socavado por los demás países, y probablemente el intento ni siquiera se hará, en nombre de la preservación de la competitividad nacional. Así, es esencial hallar una solución multilateral para mantener los objetivos fundamentales de la tributación y demás regulación. Las actividades de los mercados privados que se desarrollan en todo el mundo sólo pueden ser reguladas o gravadas por organizaciones con un alcance mundial semejante.
En el presente trabajo se ha intentado describir algunas de las maneras en que puede alcanzarse dicha gobernabilidad mundial en el ámbito de la tributación sobre los ingresos de capital. Lograr este objetivo no es fácil, dada la previsible resistencia tanto de actores privados ansiosos por seguir estando libres de impuestos como de gobiernos preocupados por conservar su capacidad soberana de fijar sus propias reglas. Pero no es imposible. Además, como la preservación de la capacidad de las naciones de gravar los ingresos de capital es esencial para concretar varias metas de crucial importancia (como las de mantener y establecer adecuados servicios públicos para los pobres), bien vale la pena intentarlo.

(Traducido del inglés)
Altshuler, R. y T. S. Newlon (1993): The effects of U.S. tax policy on the income repatriation patterns of U.S. multinational corporations, en A. Giovannini, R. Glenn y J. Slemrod (eds.), Studies in International Taxation, Chicago, Illinois, The University of Chicago Press.

Annells, J. (1998): U.K. Chancellor renews attack on EC's eurobond tax plan, Tax Notes International, vol. 17, $\mathrm{N}^{\circ} 14$, Arlington, Virginia, Tax Analysts.

Avi-Yonah, R. S. (1997): International taxation of electronic commerce, Tax Law Review, vol. 52, $\mathrm{N}^{\circ}$ 3, Nueva York, New York University School of Law. (2000): Globalization, tax competition and the fiscal crisis of the welfare state, Harvard Law Review, vol. 113, $\mathrm{N}^{\circ} 7$, Cambridge, Massachusetts, Harvard Law School.

Avi-Yonah, R. S. y L. Z. Swartz (1997): Virtual taxation: Source based taxation in the age of derivatives, The Journal of Derivatives, vol. $4, \mathrm{~N}^{\circ} 2$, Nueva York, Institutional Investor, Inc.

Banco Mundial (1994): Tax Policy Handbook, Washington, D.C.

Bond, E. (1981): Tax holidays and industry behavior, The Review of Economics and Statistics, vol. 63, Cambridge, Massachusetts, Harvard University.

Boskin, M. J. y W. G. Gale (1987): New results on the effects of tax policy on the international location of investment, en M. Feldstein (ed.), The Effects of Taxation on Capital Accumulation, Chicago, Illinois, The University of Chicago Press.

Cohen, E. S. (1998): Individual international tax planning employing equity derivatives, The Journal of Derivatives, vol. $4, \mathrm{~N}^{\circ} 4$, Nueva York, Institutional Investor, Inc.

Dunning, J. H. (1988): Explaining International Production, Londres, Unwin, Hyman.

Estados Unidos, Departamento del Tesoro (1992): Integration of the Individual and Corporate Tax Systems: Taxing Business Income Once, Washington, D.C.

Gardner, E. H. (1992): Taxes on capital income: A survey, en G. Kopits (ed.), Tax Harmonization in the European Community, Washington, D.C., Fondo Monetario Internacional (FMI).

Guisinger, S. E. y otros (1985): Investment Incentives and Performance Requirements: Patterns of International Trade, Production, and Investment, Nueva York, Praeger.
Hennart, J. F. (1991): The transaction cost theory of the multinational enterprise, en C. Pitelis y R. Sugden (eds.), The Nature of the Transnational Firm, Londres, Routledge.

Hines Jr., J. R. (1991): The flight paths of migratory corporations, Journal of Accounting, Auditing and Finance, vol. 6, $\mathrm{N}^{\circ} 4$, Boston, Warren, Gorham \& Lamont.

(1999): Lessons from behavioral responses to international taxation, National Tax Journal, vol. 52, $\mathrm{N}^{\circ} 2$, Washington, D.C., National Tax Association.

Hines Jr., J. R. y E. M. Rice (1994): Fiscal paradise: Foreign tax havens and American business, Quarterly Journal of Economics, vol. 109, $\mathrm{N}^{\circ} 1$, Cambridge, Massachusetts, Harvard University.

Intel Corporation (1998): www.intel.com/intel/intelis/sites.htm (1999): Annual Report, www.intel.com

Kant, C. (1996): Foreign Direct Investment and Capital Flight, Princeton studies in international finance, $\mathrm{N}^{\circ} 80$, Princeton, New Jersey, Princeton University.

Leibfritz, W. y otros (1995): Ageing populations, pension systems and government budgets, Working paper, $\mathrm{N}^{\circ} 156$, París, Organización de Cooperación y Desarrollo Económicos (OCDE), Departamento de Economía.

May, G. (1996): Flying on instruments: Synthetic investments and withholding tax avoidance, Tax Notes International, vol. 73, $\mathrm{N}^{\circ} 10, \mathrm{~N}^{\circ} 73$, Arlington, Virginia, Tax Analysts.

McLure Jr., C. E. (1989): U.S. tax laws and capital flight from Latin America, Inter-American Law Review, vol. 20, 으 2, Miami, Estados Unidos, Universidad de Miami.

(1996): Tax policies for the 21st century, Keynote address to the International Fiscal Association Congress, Ginebra.

Mendoza, E. G., A. Razin y L. L. Tesar (1994): Effective tax rates in macroeconomics: estimates of tax rates on factor income and consumption, Journal of Monetary Economics, vol. 34, $\mathrm{N}^{\circ} 3$, Amsterdam, Países Bajos, North-Holland Publishing Company.

Mendoza, E. G., G. M. Milesi-Ferretti y P. Asea (1996): On the Ineffectiveness of Tax Policy in Altering Long-Run Growth, Discussion paper, $\mathrm{N}^{\circ} 1378$, Londres, Centro de Investigación sobre Políticas Económicas. 
Muten, L. (1994): International experience of how taxes influence the movement of private capital, Tax Notes International, vol. 8, $\mathrm{N}^{\circ} 11$, Arlington, Virginia, Tax Analysts.

Naciones Unidas (2001): Report of the Secretary-General to the Preparatory Committee for The High-Level International Intergovernmental Event on Financing for Development, Washington, D.C.

OCDE (Organización de Cooperación y Desarrollo Económicos) (1998): Harmful Tax Competition: An Emerging Global Issue, París.

Owens, J. y J. Sasseville (1997): Emerging Issues in Tax Reform, Tax Notes International, Arlington Virginia, Tax Analysts.

Pechman, J. A. (1987): Federal Tax Policy, $5^{a}$ edición, Washington, D.C., Brookings Institution.

Peroni, R. J. (1997): Back to the future: A path to progressive reform of U.S. international income tax rules, Inter-American Law Review, vol. 51, $\mathrm{N}^{\circ}$ 4, Miami, Estados Unidos, Universidad de Miami.

PNUD (Programa de las Naciones Unidas para el Desarrollo) (1997): Informe sobre desarrollo humano 1997, Nueva York.

Razin, A. y E. Sadka (1991): International tax competition and gains from tax harmonization, Economics Letters, vol. 37, $\mathrm{N}^{\circ} 1$, Amsterdam, North Holland Co.
Rodrik, D. (1997): Has Globalization Gone Too Far?, Washington, D.C., Brookings Institution.

Sen, A. (1997): Development thinking at the beginning of the XXI century, en L. Emmerij (ed.), Economic and Social Development in the XXI Century, Washington, D.C., Banco Interamericano de Desarrollo (BID).

Subbarao, K. y otros (1997): Safety Net Programs and Poverty Reduction: Lessons from Cross-Country Experience, Washington, D.C., Banco Mundial.

Tanzi, V. (1995): Taxation in an Integrating World, Washington, D.C., Brookings Institution.

The Economist (1998): Meltdown in Russia, Londres, The Economist Newspaper Limited, 29 de agosto.

UNCTAD (Conferencia de las Naciones Unidas sobre Comercio y Desarrollo) (1996): World Investment Report 1996. Investment, Trade and International Policy Arrangements, Ginebra.

Unión Europea (1998): Conclusions of the ECOFIN Council Meeting on 1 December 1997 Concerning Taxation Policy, Luxemburgo, Oficina de Publicaciones Oficiales de las Comunidades Europeas.

Vernon, R. (1998): In the Hurricane's Eye, Cambridge, Massachusets, Harvard University Press.

Viherkentta, T. (1991): Tax Incentives in Developing Countries and International Taxation, Boston, Kluner Law International. 